Article

\title{
Highly Sensitive and Transparent Urea-EnFET Based Point-of-Care Diagnostic Test Sensor with a Triple-Gate a-IGZO TFT
}

\author{
Seong-Kun Cho (D) and Won-Ju Cho *(D)
}

Citation: Cho, S.-K.; Cho, W.-J. Highly Sensitive and Transparent Urea-EnFET Based Point-of-Care Diagnostic Test Sensor with a Triple-Gate a-IGZO TFT. Sensors 2021, 21, 4748. https://doi.org/10.3390/ s21144748

Academic Editor: Antonio Di Bartolomeo

Received: 18 June 2021

Accepted: 9 July 2021

Published: 12 July 2021

Publisher's Note: MDPI stays neutral with regard to jurisdictional claims in published maps and institutional affiliations.

Copyright: (c) 2021 by the authors. Licensee MDPI, Basel, Switzerland. This article is an open access article distributed under the terms and conditions of the Creative Commons Attribution (CC BY) license (https:/ / creativecommons.org/licenses/by/ $4.0 /)$.
Department of Electronic Materials Engineering, Kwangwoon University, 20, Gwangun-ro, Nowon-gu, Seoul 01897, Korea; whtjdrms98@gmail.com

* Correspondence: chowj@kw.ac.kr; Tel.: +82-940-5163

\begin{abstract}
In this study, we propose a highly sensitive transparent urea enzymatic field-effect transistor (EnFET) point-of-care (POC) diagnostic test sensor using a triple-gate amorphous indium gallium zinc oxide (a-IGZO) thin-film pH ion-sensitive field-effect transistor (ISFET). The EnFET sensor consists of a urease-immobilized tin-dioxide $\left(\mathrm{SnO}_{2}\right)$ sensing membrane extended gate (EG) and an a-IGZO thin film transistor (TFT), which acts as the detector and transducer, respectively. To enhance the urea sensitivity, we designed a triple-gate a-IGZO TFT transducer with a top gate (TG) at the top of the channel, a bottom gate (BG) at the bottom of the channel, and a side gate (SG) on the side of the channel. By using capacitive coupling between these gates, an extremely high urea sensitivity of $3632.1 \mathrm{mV} /$ pUrea was accomplished in the range of pUrea 2 to 3.5; this is 50 times greater than the sensitivities observed in prior works. High urea sensitivity and reliability were even obtained in the low pUrea ( 0.5 to 2 ) and high pUrea (3.5 to 5) ranges. The proposed urea-EnFET sensor with a triple-gate a-IGZO TFT is therefore expected to be useful for POC diagnostic tests that require high sensitivity and high reliability.
\end{abstract}

Keywords: ion-sensitive field-effect transistor; triple-gate; extended gate; capacitive coupling; urea sensor; antigen-antibody

\section{Introduction}

Urea sensors are utilized in various fields, such as food [1,2], environmental protection [3,4], pharmaceuticals, fertilizers, and especially bio-clinical analysis [5]. In the human body, urea is the main end-product of nitrogen metabolism, and it is mainly removed from the body in urine but is also secreted in body fluids such as blood, sweat, and saliva. Therefore, we can diagnose and/or treat pathologies such as renal insufficiency, hyperpyrexia, leukemia, diarrheal diseases, diabetes mellitus, and hyperthyroidism by identifying changes in the urea concentration of the blood, serum, or urine [6]. In general, the level of urea in serum ranges from $2.5 \mathrm{mM}$ to $7.5 \mathrm{mM}$ and rapidly increases under pathophysiological conditions, providing key information for the diagnosis of renal function and various kidney and liver diseases. Chronic kidney disease or end-stage renal disease is generally caused by a progressive loss of kidney function; this increases the level of urea in the blood, which is known as azotemia or uremia. At urea levels above $30 \mathrm{mM}$, renal insufficiency becomes important, and hemodialysis is required [7]. Therefore, it is crucial to develop new technologies to assess urea levels quickly and at a low cost.

The ion-sensitive field-effect transistor (ISFET), first introduced by Bergveld in the early 1970s [8], has been used extensively for physiological measurements in biomedical applications. Because ISFETs have a metal-oxide-semiconductor FET (MOSFET) device structure, they can be manufactured using the complementary metal-oxide-semiconductor (CMOS) process, which is the most promising technology for microsystems. Unlike MOSFETs, however, ISFETs do not have metal or polysilicon gate electrodes, which exposes the 
gate oxide insulator to the electrolyte solution. In an ISFET, the gate voltage is supplied to the reference electrode immersed in the same electrolyte solution, instead of to the gate electrode, as in a MOSFET. Thus, the gate potential, which modulates the drain current, is defined by the voltage supplied by the reference electrode and the surface potential of the sensing membrane. As indicated by the site binding model, the surface potential of the sensing membrane is determined by the ions adsorbed on the gate insulator surface of the inner Helmholtz plane in the Stern-Gouy-Chapman model of the electrical double layer $[9,10]$. Semiconductor-based ISFETs are compatible with the CMOS manufacturing processes, enabling mass production and miniaturization. Conversely, the gate insulators (i.e., sensing membranes) of an ISFET are susceptible to damage by the chemical ions in the electrolyte solution [11]. Therefore, an ISFET with an immobilized enzyme extended gate (EG) was proposed by Clark and Lyons and introduced as the urea-enzymatic FET (EnFET) $[12,13]$. In the EnFET, the detection of urea is accomplished through a bio-signal delivered to the $\mathrm{pH}$-ISFET sensor platform via a reaction with urease enzymes immobilized on the surface of the EG sensing membrane. Figure 1 shows the sensing mechanism of the urease enzymes immobilized $\mathrm{SnO}_{2}$ sensing membrane. Since $\mathrm{SnO}_{2}$ sensing membrane without immobilized urease does not react with urea, the surface potential does not change. On the other hand, since the urease-immobilized $\mathrm{SnO}_{2}$ sensing membrane reacts with urea and forms a reaction product, the surface potential changes. Urea hydrolysis is catalyzed by urease, as described in the following expression:

$$
\left(\mathrm{NH}_{2}\right)_{2} \mathrm{CO}+3 \mathrm{H}_{2} \mathrm{O} \stackrel{\circ}{\stackrel{\text { urease }}{\rightarrow}} \mathrm{CO}_{2}+2 \mathrm{NH}_{4}^{+}+2 \mathrm{OH}^{-} \text {. }
$$

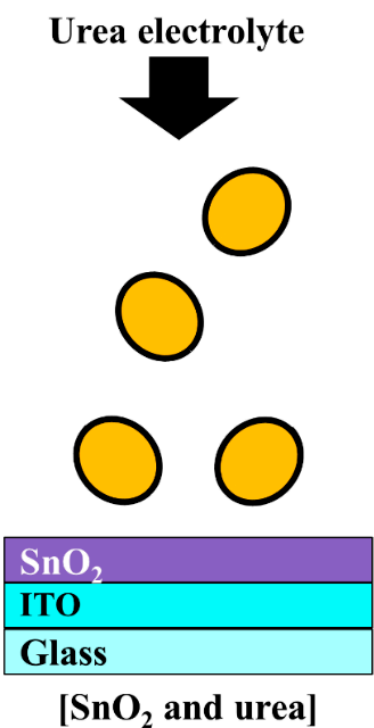

(a)

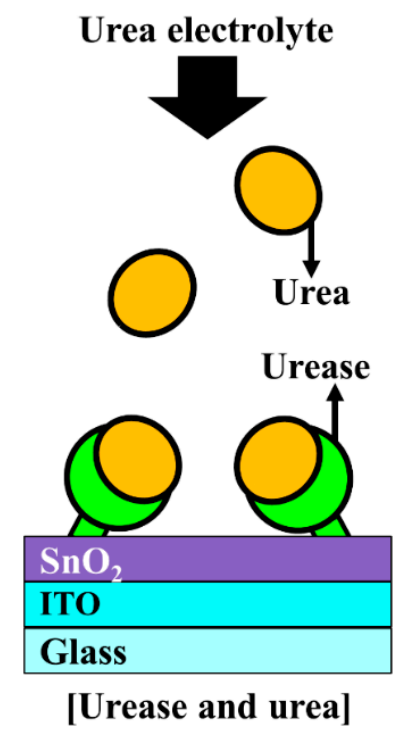

(b)

Figure 1. Schematic illustration of (a) the interaction between the $\mathrm{SnO}_{2}$ membrane and urea and (b) the interaction between urease and urea.

The reaction product increases the $\mathrm{pH}$ at the surface of the sensing membrane. If the urease enzyme is not immobilized, there is no change in the surface potential because there is no reaction product. The potential change between the sensing membrane surface and the urea solution allows the pH-ISFET sensor platform to indirectly determine the urea concentration by calibrating the change in the major parameters of the EnFET in the sensing operation [13]. For this reason, the pH-ISFET sensor platform can be applied as a urea-EnFET. However, the sensitivity of the conventional $\mathrm{pH}$-ISFET sensor platform has a Nernst limit $(59.5 \mathrm{mV} / \mathrm{pH}$ at room temperature) that is not suitable for detecting weak biological signals. This poor sensitivity constrains the detection of biomaterials with small 
concentrations and low amplitude biological signals in the human body, such as DNA, cells, antigen antibodies, enzymes, and hormones [14-17].

In this study, we overcome the fundamental chemical damage problem of the gate insulator in an FET transducer by constructing a urea-EnFET sensor with an EG that is separate from the FET transducer. This configuration is expected to produce a cost-effective sensor device because the low-cost EG (disposable detector) can easily be exchanged, thereby allowing the continued use of expensive and sophisticated FET transducers without damage. To implement a transparent urea-EnFET sensor, we fabricated a transducer unit based on a triple-gate amorphous indium gallium zinc oxide (a-IGZO) thin film transistor (TFT) on a glass substrate. In addition, we fabricated a sensing unit by urease immobilization through surface treatment on $\mathrm{SnO}_{2}$ sensing membrane EG. We implemented the urea-EnFET sensor by electrically connecting triple-gate a-IGZO TFTs and urease-immobilized $\mathrm{SnO}_{2}$ sensing membrane EG. Therefore, we achieved increased sensitivity to weak signal biomaterials in the a-IGZO TFT design with a triple-gate structure that consists of a top gate (TG), bottom gate (BG), and side gate (SG) at the top, bottom, and side of the TFT channel, respectively. Capacitive coupling occurs between these triple gates, allowing significantly amplified sensitivity. By applying this unique pH-ISFET sensor platform to the urea-EnFET, we achieved a very high urea sensitivity of $3632.1 \mathrm{mV} / \mathrm{pUrea}$ in the range of pUrea 2 to 3.5, which is 50 times greater than that reported in previous studies. Moreover, we demonstrate that high urea sensitivity is reliably obtained, even in the low pUrea range (0.5 to 2 ) and the high pUrea range (3.5 to 5). Accordingly, the urea-EnFET sensor platform proposed in this paper is a useful portable sensing tool for urea detection, and it is expected to serve as a highly sensitive sensor platform for point-of-care (POC) diagnostic tests for the detection of a variety of biomaterials, such as DNA, cells, antigen antibodies, enzymes, and hormones.

\section{Materials and Methods}

\subsection{Fabrication Process of the Triple-Gate a-IGZO TFT Transducer Unit}

A $1 \times 1 \mathrm{~cm}^{2}$ transparent glass substrate (Corning Inc., New York, NY, USA) was used as the substrate of the a-IGZO TFT. To fabricate the triple-gate a-IGZO TFT transducer, a 300-nm-thick indium tin oxide (ITO) layer and a 300-nm-thick $\mathrm{SiO}_{2}$ film were deposited sequentially as the BG and the bottom gate insulator, respectively, using an RF magnetron sputtering system. ITO was deposited at a deposition power of $100 \mathrm{~W}$, a working pressure of 3 mTorr in an ambient of Ar with a flow rate of $20 \mathrm{sccm}$, and $\mathrm{SiO}_{2}$ was deposited at a deposition power of $200 \mathrm{~W}$, a working pressure of 3 mTorr in an ambient of $\mathrm{Ar} / \mathrm{O}_{2}$ with a flow rate of 30/2 sccm. Then, a 20-nm-thick a-IGZO (In:Ga:Zn = 1:1:1) film was deposited by the RF magnetron sputtering system at a deposition power of $100 \mathrm{~W}$, a working pressure of $6 \mathrm{mTorr}$ in an ambient of Ar with a flow rate of $30 \mathrm{sccm}$ as the active region. The active channel region (width/length $=20 / 10 \mu \mathrm{m}$ ) was defined using photolithography and wet etching processes. To form the source/drain (S/D), a 100-nm-thick ITO layer was deposited by an RF magnetron sputtering system and a lift-off process. Subsequently, a 10/35-nm-thick $\mathrm{SiO}_{2} / \mathrm{Ta}_{2} \mathrm{O}_{5}$ stacked top gate insulator was deposited on the active layer using RF magnetron sputtering system. $\mathrm{SiO}_{2}$ was deposited at a deposition power of $200 \mathrm{~W}$, a working pressure of 3 mTorr in an ambient of $\mathrm{Ar} / \mathrm{O}_{2}$ with a flow rate of $30 / 2$ sccm, and $\mathrm{Ta}_{2} \mathrm{O}_{5}$ was deposited at a deposition power of $75 \mathrm{~W}$, a working pressure of $3 \mathrm{mTorr}$ in an ambient of Ar with a flow rate of $20 \mathrm{sccm}$. The $\mathrm{SiO}_{2} / \mathrm{Ta}_{2} \mathrm{O}_{5}$ stacked top gate insulator increases the capacitive coupling with the capacitance of the bottom gate oxide and reduces the gate leakage current. After the deposition of a 150-nm-thick ITO film by RF magnetron sputtering, the TG and SG were formed simultaneously using photolithography and lift-off processes. Finally, to improve the contact characteristics of the gate electrodes, post-deposition annealing was performed at $250{ }^{\circ} \mathrm{C}$ for $30 \mathrm{~min}$ in $\mathrm{O}_{2}$ ambient using a resistive heating furnace. A schematic diagram of the fabricated triple-gate a-IGZO TFT is shown in Figure 2a, and optical microscopy and photographic images of the device are shown in Figures $2 b$ and $2 c$, respectively. 


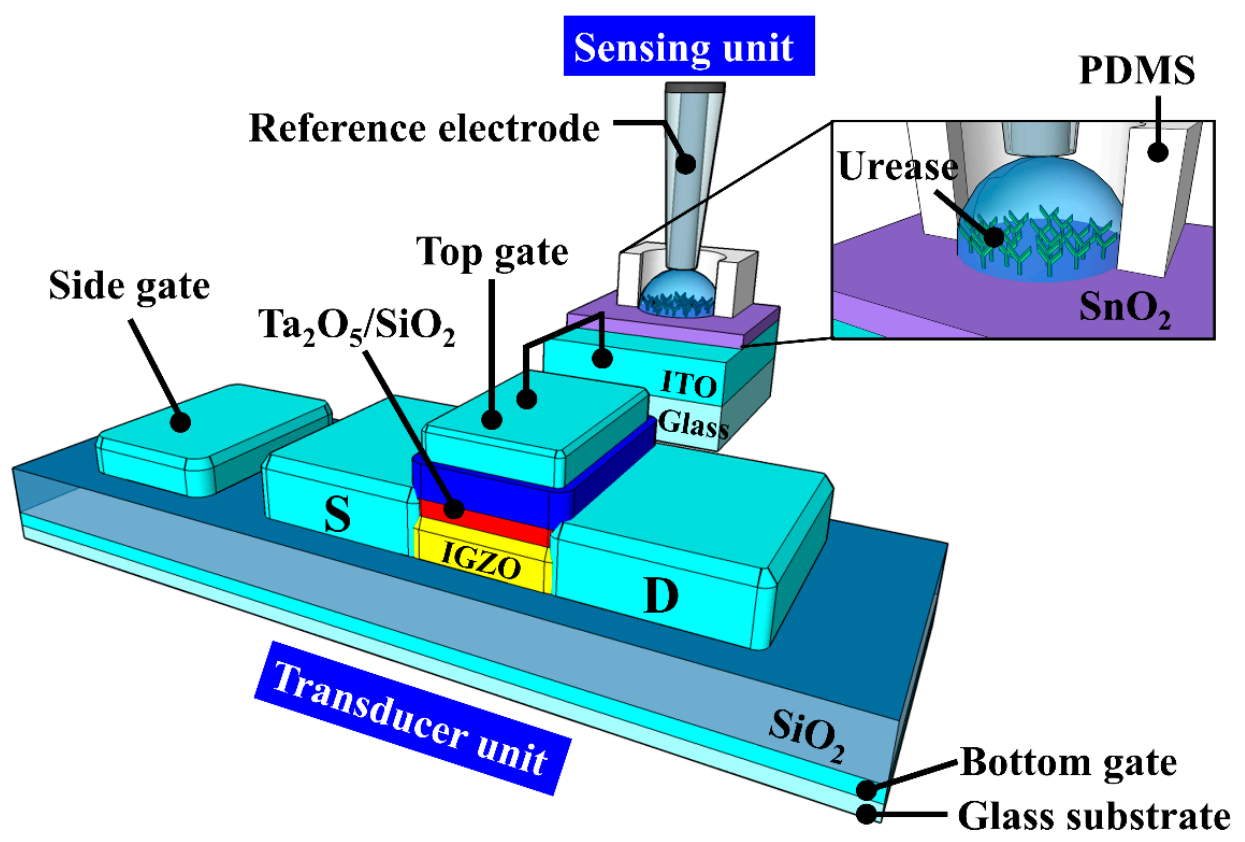

(a)

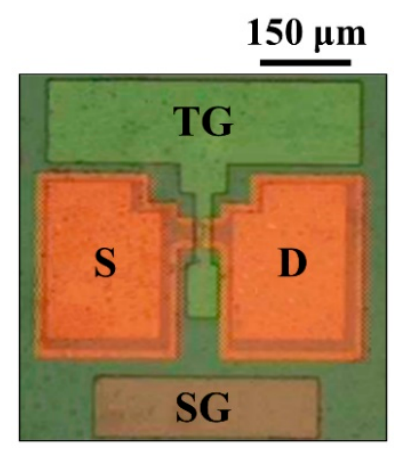

(b)

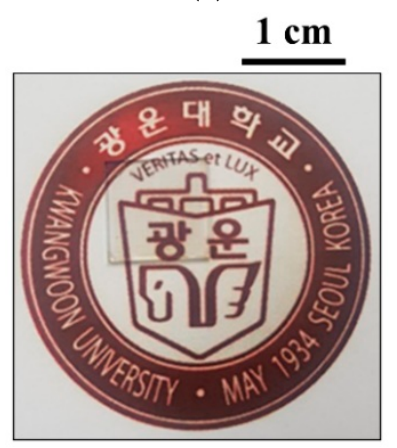

(c)

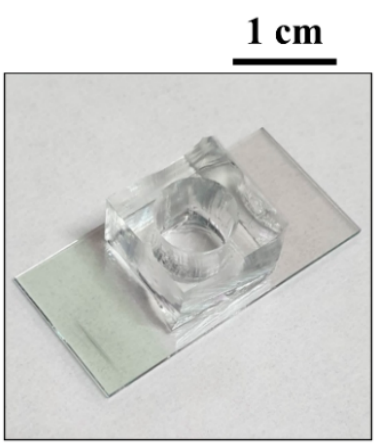

(d)

Figure 2. (a) Schematic diagram of the urea-EnFET sensor configured by the triple-gate a-IGZO TFT and urease-immobilized $\mathrm{SnO}_{2}$ sensing membrane EG; (b) optical microscopy and (c) photographic images of the fabricated triple-gate a-IGZO TFT; and (d) photographic image of the urease-immobilized $\mathrm{SnO}_{2}$ sensing membrane EG.

\subsection{Fabrication Process of the Urease-Immobilized $\mathrm{SnO}_{2}$ Sensing Membrane EG Sensing Unit}

To fabricate the urease-immobilized $\mathrm{SnO}_{2}$ sensing membrane EG sensing unit, a 300-nm-thick ITO electrode layer and a 50-nm-thick $\mathrm{SnO}_{2}$ sensing membrane were sequentially deposited on a $1.5 \times 2.5 \mathrm{~cm}^{2}$ transparent glass substrate (Corning Inc., New York, NY, USA) using RF magnetron sputtering system. $\mathrm{SnO}_{2}$ was deposited at a deposition power of $100 \mathrm{~W}$, a working pressure of 3 mTorr in an ambient of Ar with a flow rate of $30 \mathrm{sccm}$. Then, covalent bonding was used to immobilize urease on the $\mathrm{SnO}_{2}$ sensing membrane of the EG. Figure 3a is a schematic of the covalent bonding process used to immobilize urease enzymes on the $\mathrm{SnO}_{2}$ sensing membrane of the EG. The covalent bonding process consists of four steps: (1) the $\mathrm{SnO}_{2}$ surface of the EG was treated with $\mathrm{O}_{2}$ plasma to generate hydroxyl groups; (2) the EGs were silylated by immersion in a $40{ }^{\circ} \mathrm{C}$, 9\% 3-aminopropyltriethoxysilane (APTS) solution for $4 \mathrm{~h}$; (3) the samples were placed in $10 \%$ glutaraldehyde (GA), a dual function group for crosslinking urease amines and APTS, for $1 \mathrm{~h}$; and (4) the urease powder was mixed with the phosphate buffer solution (PBS) at a concentration of $1.5 \mu \mathrm{g} / \mathrm{mL}$, and the sample surfaces were soaked in the urease solution. The EG specimens were then stored overnight in a refrigerator at $4{ }^{\circ} \mathrm{C}$ to immobilize the urease enzymes, after which the immobilized enzyme was rinsed with PBS. After being rinsed with PBS, a $0.6 \mathrm{~cm}$ diameter polydimethylsiloxane (PDMS) reservoir for urea elec- 
trolyte storage was fixed on the surface-treated $\mathrm{SnO}_{2}$. All the reagents were purchased from Sigma Aldrich Co. (St. Louis, MO, USA) and used without further purification. The urease-immobilized $\mathrm{SnO}_{2}$ sensing membrane EG is connected to the dark box through an electrical cable, which is connected to the top gate of the triple-gate a-IGZO TFT through a positioner. Figure 2a is a schematic diagram showing the electrical connection between the fabricated urease-immobilized $\mathrm{SnO}_{2}$ sensing membrane EG and triple-gate a-IGZO TFTs, and a photographic image of the fabricated EG is shown in Figure 2d.

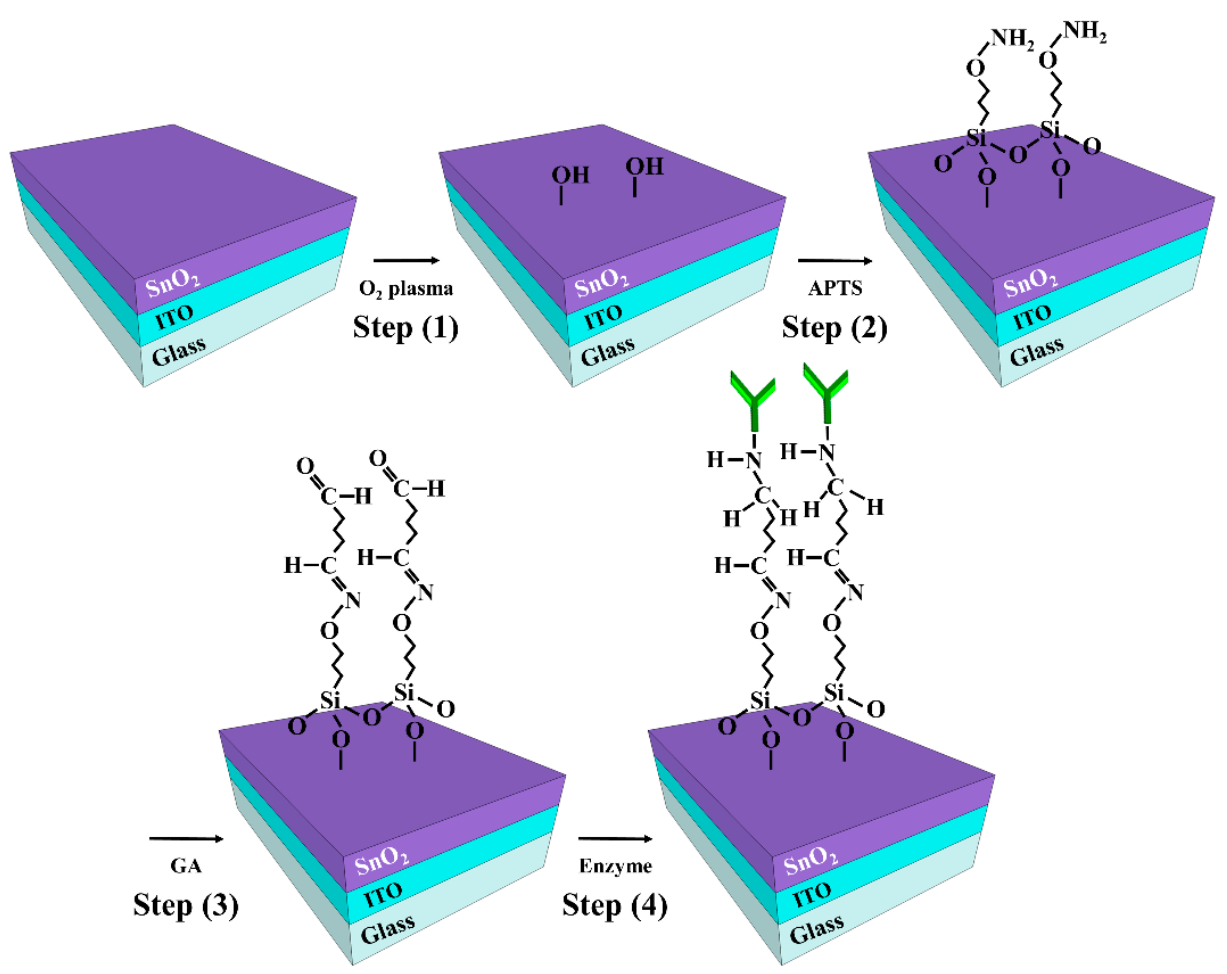

(a)

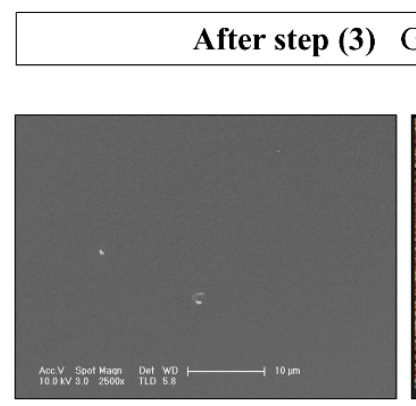

(b)

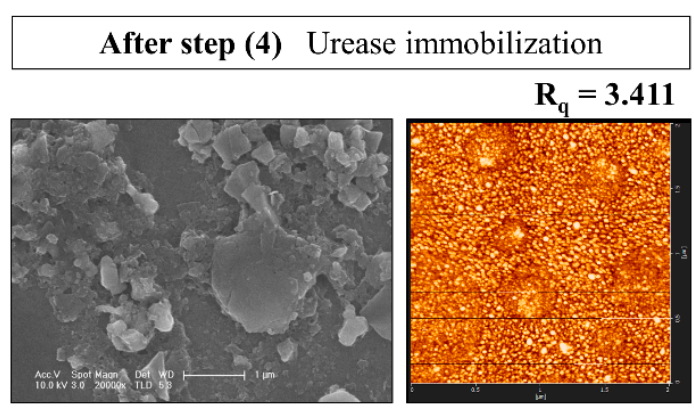

(c)

Figure 3. (a) Schematic diagram of the covalent bond process on the $\mathrm{SnO}_{2}$ sensing membrane of the EG. SEM and AFM images of the $\mathrm{SnO}_{2}$ membrane surface after (b) Step 3 (GA) and (c) Step 4 (immobilization of the urease enzymes).

To verify the immobilization of the urease enzymes, the surface morphology of the $\mathrm{SnO}_{2}$ membranes was analyzed. Atomic force microscopy (AFM; SPM Solver-Pro, NTMDT, Moscow, Russia) and scanning electron microscopy (SEM; Sirion 400, FEI Company, Hillsboro, OR, USA) images of the $\mathrm{SnO}_{2}$ membrane surfaces after the GA procedure and urease enzyme immobilization are shown in Figure 3b,c. After the third surface treatment, the GA layer undergoes automatic polymerization on the $\mathrm{SnO}_{2}$ surface and forms large domains. The aldehyde groups in GA can be used to bind urease enzymes covalently through the exposed amine groups of lysine amino acids. SEM images taken after urease enzyme immobilization confirm the presence of crystal structures over and throughout the 
$\mathrm{SnO}_{2}$ membrane surface, and AFM images show that the root-mean-square roughness $\left(\mathrm{R}_{\mathrm{q}}\right)$ of the surface increased significantly from $0.5804 \mathrm{~nm}$ to $3.411 \mathrm{~nm}$.

\subsection{Device Characterization}

The current-voltage characteristics of the triple-gate a-IGZO TFT-based ISFET sensor platform were measured using an Agilent 4156B precision semiconductor parameter analyzer. The $\mathrm{Ag} / \mathrm{AgCl}$ reference electrode (Horiba 2080A-06T) was used for $\mathrm{pH}$ and urea sensing. All measurements were conducted in a dark box to avoid interference from external light and noise. We evaluated the $\mathrm{pH}$ sensitivity in the TG, BG, and SG modes. We then examined the reliability and stability by measuring the hysteresis and drift effects operating between the $\mathrm{pH}$ electrolytes and the sensing membrane. After evaluating the $\mathrm{pH}$ sensing performance of the triple-gate a-IGZO TFT-based ISFET sensor platform, the urea sensitivity was evaluated in the TG, BG and SG modes.

The proposed triple-gate a-IGZO TFT-based ISFET sensor platform is capable of detection in three operating modes, which are shown in Figure 4: (a) TG-biasing TGsensing (TG mode), (b) BG-biasing TG-sensing (BG mode), and (c) SG-biasing TG-sensing (SG mode). The TG mode functions like a conventional ISFET, where the BG electrode is grounded and measures the drain current while sweeping the TG bias voltage is delivered through the reference electrode, as shown in Figure 4a. In contrast, the BG mode measures the drain current while grounding the TG electrode and sweeping the BG bias voltage, as shown in Figure $4 \mathrm{~b}$. The SG mode measures the drain current while sweeping the SG bias voltage by grounding the TG and floating the BG, as shown in Figure 4c. In particular, BFG in Figure $4 \mathrm{c}$ refers to the BG in a floating state. Table 1 summarizes the bias conditions for each gate voltage in the three operating modes of the triple-gate a-IGZO TFT-based ISFET sensor platform.

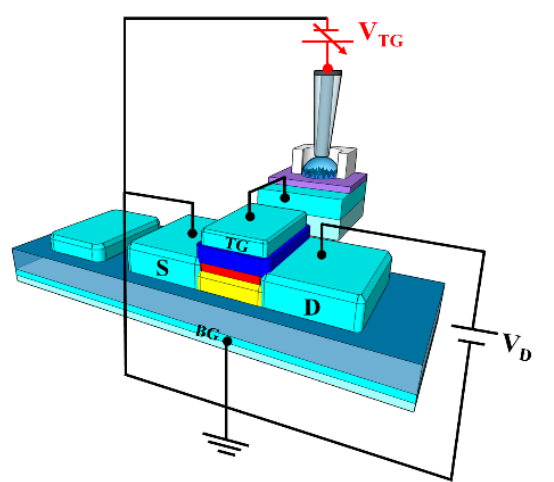

(a)

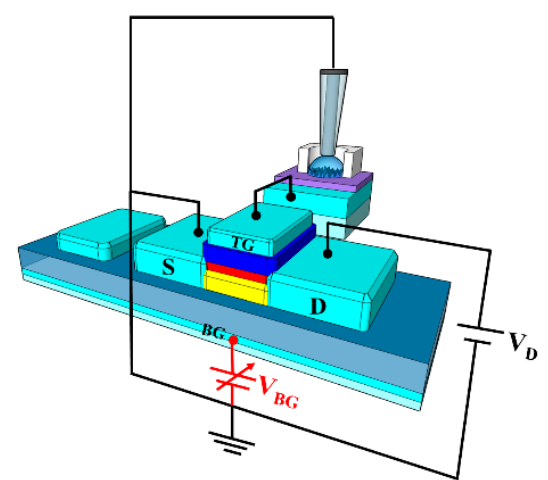

(b)

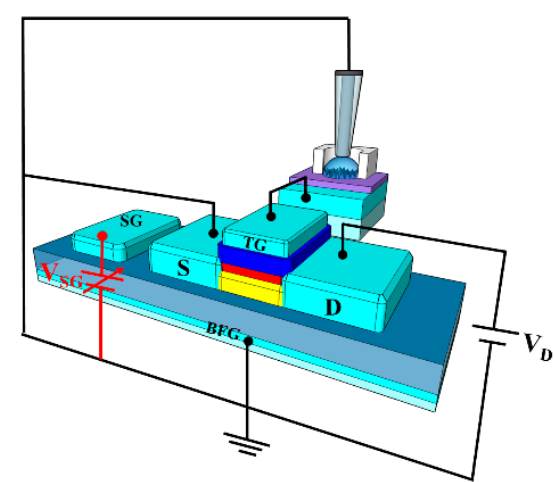

(c)

Figure 4. Schematics of the three different sensing modes of the proposed triple-gate a-IGZO TFT-based ISFET sensor platform: (a) TG-biasing TG-sensing (TG mode), (b) BG-biasing TG-sensing (BG mode), and (c) SG-biasing TG-sensing (SG mode).

Table 1. Bias conditions for each gate voltage in the three operating modes of the triple-gate a-IGZO TFT-based sensor platform.

\begin{tabular}{cccc}
\hline & TG Mode & BG Mode & SG Mode \\
\hline TG & Sweep & Ground & Ground \\
\hline BG & Ground & Sweep & Floating \\
\hline SG & NA & NA & Sweep \\
\hline
\end{tabular}

Figure 5 shows (a) a cross-sectional schematic and (b) an equivalent circuit of a triplegate a-IGZO TFT-based ISFET sensor platform. The black, red, and blue dotted boxes in Figure 5 correspond to the TG, BG, and SG modes of the ISFET sensor, respectively. 


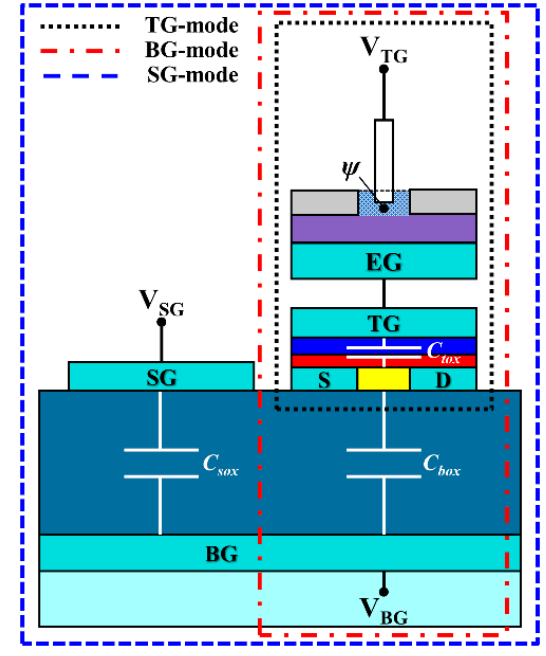

(a)

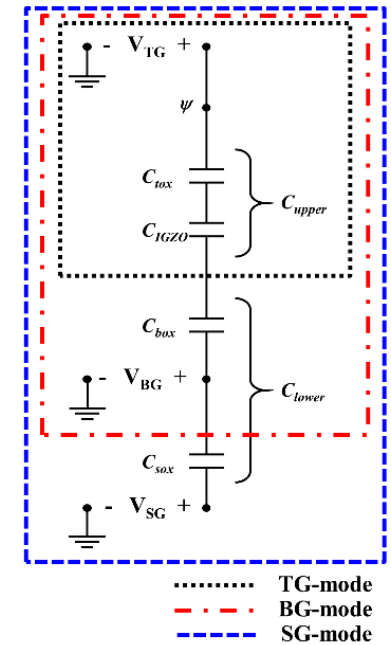

(b)

Figure 5. (a) Cross-sectional diagram and (b) equivalent circuit of a triple-gate a-IGZO TFT-based ISFET sensor platform.

In general, conventional ISFETs driven by the TG mode have a sensitivity limit (ideal Nernst response at $300 \mathrm{~K}$ of $59.5 \mathrm{mV} / \mathrm{pH}$ ) according to the site binding model [18]. This is because the threshold voltage of the TG $\left(V_{T G}^{\text {th }}\right)$ is driven by changes in the TG mode, in accordance with the surface potential $(\psi)$ of the sensing membrane from the electrolyte:

$$
\Delta V_{t h}^{T G}=-\Delta \psi
$$

where $\Delta V_{t h}^{T G}$ is the threshold voltage shift of the TG.

In contrast, in the BG mode, the sensitivity can be amplified by the capacitive coupling effect between the top and bottom of the TFT channel. Thus, the threshold voltage of the $\mathrm{BG}\left(V_{t h}^{B G}\right)$ driven by the BG mode is given not only by $\psi$ from electrolyte but also by the capacitance ratio between $C_{\text {upper }}$ and $C_{\text {lower }}$. In this mode, $C_{\text {upper }}$ is a series combination of the TG capacitance $C_{t o x}$ and the channel depletion capacitance $C_{I G Z O}$, while $C_{\text {lower }}$ is the BG capacitance $C_{b o x}$. Then, the sensitivity of the BG mode according to the BG voltage sweep is given by Equation (2) [19]:

$$
\Delta V_{t h}^{B G}=-\frac{C_{\text {upper }}}{C_{\text {lower }}} \Delta \psi=\frac{C_{I G Z O} C_{t o x}}{C_{b o x}\left(C_{I G Z O}+C_{t o x}\right)} \Delta V_{t h}^{T G},
$$

where $\Delta V_{t h}^{B G}$ is the threshold voltage shift of the BG. This means that the $\psi$ of the sensing membrane is transferred to the TG and results in $\Delta V_{t h}^{B G}$ being shifted in proportion to $C_{t o x} / C_{b o x}$. Thus, for the BG mode, a thicker BG oxide and thinner TG oxide are more desirable to further enhance the sensitivity by increasing the capacitive coupling ratio. This also implies that TG insulator engineering using high-k materials is effective for increasing $C_{\text {upper }}$ while reducing the leakage current, thereby improving the sensitivity. Nevertheless, increasing the thickness of the BG oxide entails a longer manufacturing process time and higher cost, while thinner TG oxides cause higher leakage currents, making this an inefficient approach. In the device proposed in this study, $C_{I G Z O}, C_{t o x}$, and $C_{b o x}$ are about $1.416 \mathrm{pF}, 0.49 \mathrm{pF}$, and $0.023 \mathrm{pF}$, respectively, and substituting them into Equation (2), the theoretical amplification ratio of BG mode is about 16.

Meanwhile, the proposed triple-gate a-IGZO TFT-based ISFET sensor platform has an excellent SG mode for sensitivity amplification. We introduced the SG mode to increase the amplification ratio by further reducing the $C_{\text {lower }}$. In this case, the $C_{\text {lower }}$ of the device is $\left(C_{b o x} \times C_{s o x}\right) /\left(C_{b o x}+C_{s o x}\right)$ because the floated BG connects $C_{b o x}$ and $C_{s o x}$ in series. $C_{s o x}$ is the capacitance between the SG and BG and results in the SG gate effectively doubling the thickness of the BG oxide [20]. Therefore, since $C_{\text {lower }}$ is smaller in SG mode than in BG 
mode, SG mode is amplified more than BG mode. For the SG mode using an SG voltage sweep, the capacitive coupling and sensitivity are described in Equation (3) [21]:

$$
\Delta V_{\text {th }}^{S G}=-\frac{C_{\text {upper }}}{C_{\text {lower }}} \Delta \psi=\frac{C_{I G Z O} C_{t o x}\left(C_{b o x}+C_{\text {sox }}\right)}{\left(C_{I G Z O}+C_{t o x}\right)\left(C_{b o x} C_{s o x}\right)} \Delta V_{t h}^{T G},
$$

where $\Delta V_{t h}^{S G}$ is the threshold voltage shift of the SG. In this mode, $C_{u p p e r}$ is a series combination of $C_{\text {tox }}$ and $C_{I G Z O}$, and $C_{\text {lower }}$ is a series combination of $C_{b o x}$ and $C_{s o x}$. In the device proposed in this study, $C_{\text {sox }}$ is about $0.015 \mathrm{pF}$, and the theoretical amplification ratio of SG mode is about 40 . This suggests that the sensitivity can be further amplified by using the SG mode of triple-gate a-IGZO TFTs.

\section{Results}

\subsection{Electrical Characteristics of the Triple-Gate a-IGZO TFT}

Figure 6 shows the typical electrical characteristics of triple-gate a-IGZO TFTs operating under TG, BG, and SG bias voltage sweep conditions corresponding to the TG, BG, and SG modes of the ISFETs, respectively. The transfer characteristic curves were measured at a drain voltage of $1 \mathrm{~V}$, and the gate voltage was swept from -0.5 to $1.0 \mathrm{~V}$ for TG mode, from -4 to $10 \mathrm{~V}$ for BG mode, and from -10 to $25 \mathrm{~V}$ using the double-sweep mode to evaluate hysteresis characteristics. Meanwhile, the output characteristics were measured from $\mathrm{V}_{\mathrm{TG}}-\mathrm{V}_{\mathrm{TH}}=0 \sim 0.5 \mathrm{~V}$ in $0.05 \mathrm{~V}$ steps for the TG mode, $\mathrm{V}_{\mathrm{BG}}-\mathrm{V}_{\mathrm{TH}}=0 \sim 5 \mathrm{~V}$ in $0.5 \mathrm{~V}$ steps for the $\mathrm{BG}$ mode, and $\mathrm{V}_{\mathrm{SG}}-\mathrm{V}_{\mathrm{TH}}=0 \sim 15 \mathrm{~V}$ in $1.5 \mathrm{~V}$ steps for the $\mathrm{SG}$ mode. In the transfer characteristic curves and the output characteristic curves of each mode, it can be seen that the drain current is adjusted according to the drain voltage and the gate voltage, respectively.

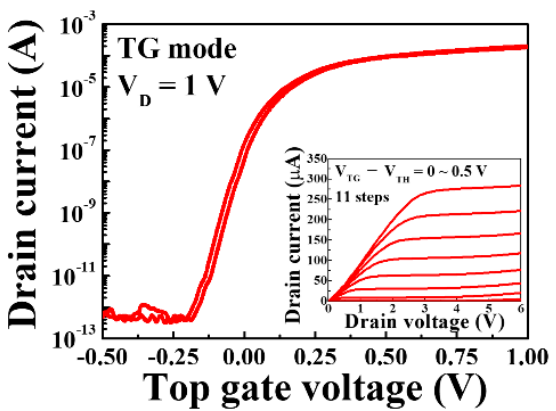

(a)

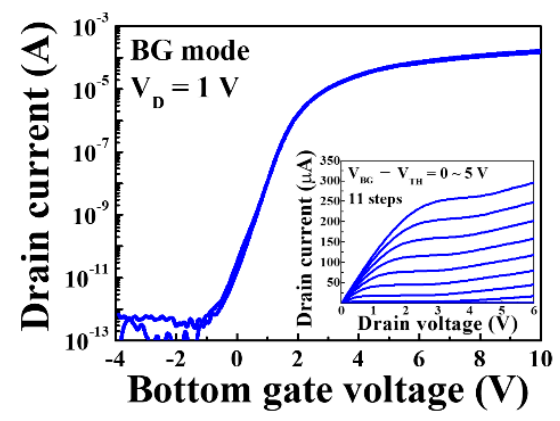

(b)

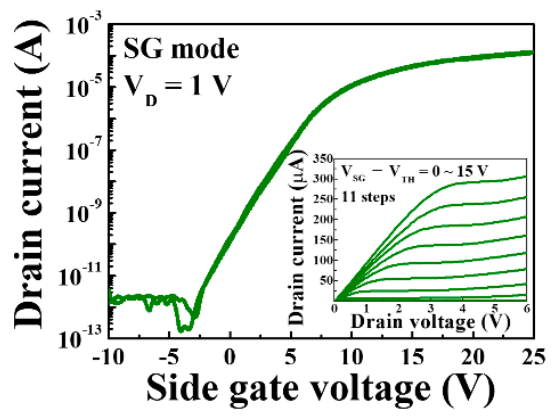

(c)

Figure 6. Transfer characteristic curves of triple-gate a-IGZO TFTs operating in the (a) TG, (b) BG, and (c) SG modes. The insets show the output characteristic curves corresponding to each operating mode.

Table 2 summarizes the electrical parameters extracted from the transfer characteristic curves. A threshold voltage $\left(\mathrm{V}_{\mathrm{TH}}\right)$ of $-0.11 \mathrm{~V}$, hysteresis voltage $\left(\mathrm{V}_{\mathrm{HYS}}\right)$ of $0.02 \mathrm{~V}$, field-effect mobility ( $\mu_{\mathrm{FE}}$ ) of $7.37 \mathrm{~cm}^{2} / \mathrm{V} \cdot \mathrm{s}$, subthreshold swing (SS) of $157.07 \mathrm{mV} / \mathrm{dec}$, and on/off current ratio $\left(\mathrm{I}_{\mathrm{ON}} / \mathrm{I}_{\mathrm{OFF}}\right)$ of $6.78 \times 10^{7}$ were obtained under top gate operation. Meanwhile, $\mathrm{V}_{\mathrm{TH}}, \mathrm{V}_{\mathrm{HYS}}, \mu_{\mathrm{FE}}, \mathrm{SS}$, and $\mathrm{I}_{\mathrm{ON}} / \mathrm{I}_{\mathrm{OFF}}$ of $0.13 \mathrm{~V}, 0.12 \mathrm{~V}, 12.89 \mathrm{~cm}^{2} / \mathrm{V} \cdot \mathrm{s}, 356.90 \mathrm{mV} / \mathrm{dec}$, and $5.62 \times 10^{7}$ were obtained under bottom gate operation, and $\mathrm{V}_{\mathrm{TH}}, \mathrm{V}_{\mathrm{HYS}}, \mu_{\mathrm{FE}}, \mathrm{SS}$ and $\mathrm{I}_{\mathrm{ON}} / \mathrm{I}_{\mathrm{OFF}}$ of $-0.28 \mathrm{~V}, 0.27 \mathrm{~V}, 19.38 \mathrm{~cm}^{2} / \mathrm{V} \cdot \mathrm{s}, 434.13 \mathrm{mV} / \mathrm{dec}$, and $4.48 \times 10^{7}$ were obtained under side gate operation. The TG, BG, and SG sweep operations of the fabricated triple-gate a-IGZO TFT all show excellent electrical characteristics, meaning that they are satisfactory as sensors for sensing $\mathrm{pH}$ or urea. 
Table 2. Electrical parameters of triple-gate a-IGZO TFTs under top gate, bottom gate, and side gate operations.

\begin{tabular}{cccccc}
\hline Operation Mode & $\mathbf{V}_{\mathrm{TH}}(\mathrm{V})$ & $\mathrm{V}_{\mathrm{HYS}}(\mathrm{V})$ & $\mu_{\mathrm{FE}}\left(\mathrm{cm}^{2} / \mathrm{V} \cdot \mathbf{s}\right)$ & $\mathrm{SS}(\mathrm{mV} / \mathrm{dec})$ & $\mathrm{I}_{\mathrm{ON}} / \mathrm{I}_{\mathrm{OFF}}(\mathrm{A} / \mathrm{A})$ \\
\hline TG & -0.11 & 0.02 & 7.37 & 157.07 & $6.78 \times 10^{7}$ \\
\hline BG & 0.13 & 0.12 & 12.89 & 356.90 & $5.62 \times 10^{7}$ \\
\hline SG & -0.28 & 0.27 & 19.38 & 434.13 & $4.48 \times 10^{7}$ \\
\hline
\end{tabular}

\section{2. $p H$ Sensing Characteristics of the Triple-Gate a-IGZO TFT-Based pH-ISFET Sensor Platform}

Figure 7 shows the transfer characteristic curves and $\mathrm{pH}$ sensitivities of the triplegate a-IGZO TFT-based $\mathrm{pH}$-ISFET sensor platform for various $\mathrm{pH}$ concentrations in the TG, BG, and SG modes. The measurements were taken using the EG with an $\mathrm{SnO}_{2}$ membrane without immobilized urease. The reference voltage for determining the shift of the threshold voltage with respect to the $\mathrm{pH}$ concentration was defined as each gate voltage at the reference drain current of $1 \mathrm{nA}\left(\mathrm{I}_{\text {ref }}\right)$. From the transfer characteristic curves, it is evident that reference voltage increases for $\mathrm{pH}$ concentrations ranging from $\mathrm{pH} 3.07$ to 9.87. The increase in the total reference voltage in this $\mathrm{pH}$ range is $0.39 \mathrm{~V}$ for the TG mode, $7.14 \mathrm{~V}$ for the BG mode, and $16.23 \mathrm{~V}$ for the SG mode, while the $\mathrm{pH}$ sensitivity, which corresponds to the slope of the linear fits, is $55.58,1038.89$, and $2363.9 \mathrm{mV} / \mathrm{pH}$ for the TG, BG, and SG modes, respectively. These results show that compared to the TG mode, the BG mode is amplified by 18.7 times and the SG mode by 42.5 times, demonstrating that the proposed sensor platform can greatly amplify the $\mathrm{pH}$ sensitivity according to the sensing mode, as shown in Equations (2) and (3).

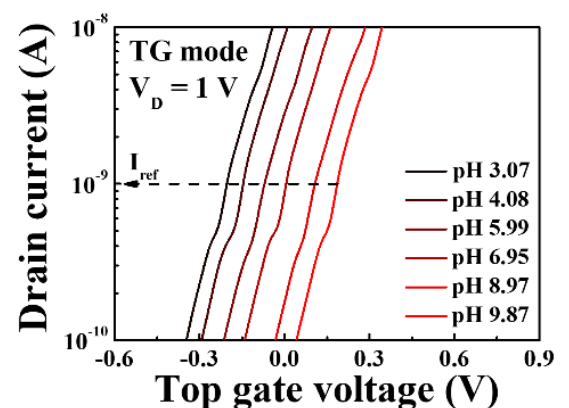

(a)

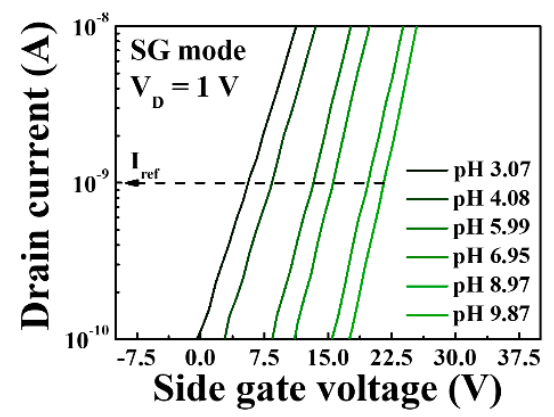

(c)

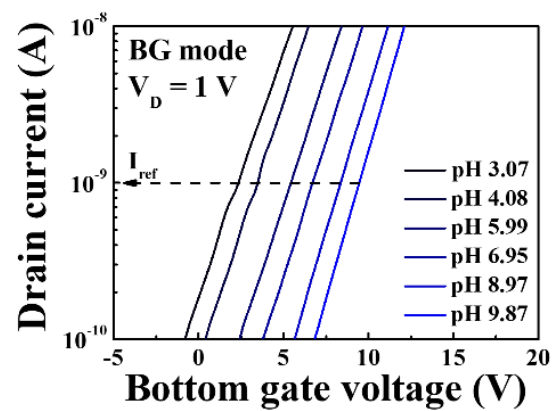

(b)

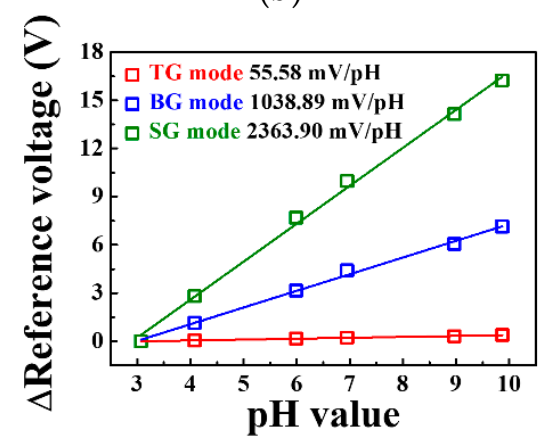

(d)

Figure 7. Transfer characteristic curves of the triple-gate a-IGZO TFT-based pH-ISFET sensor platform in (a) TG mode, (b) BG mode, and (c) SG mode. (d) Change in the reference voltage of the triple-gate a-IGZO TFT-based pH-ISFET sensor platform as a function of $\mathrm{pH}$.

Figure 8 shows the stability and reliability test of the triple-gate a-IGZO TFT-based $\mathrm{pH}$-ISFET sensor platform as determined by measuring the hysteresis voltage and drift rate. The hysteresis voltage shown in Figure $8 \mathrm{a}$ is caused by the micro potential charge of the sensing membrane, which occurs when the ions in the $\mathrm{pH}$ electrolyte react slowly with 
the sensing membrane [22]. We defined the hysteresis voltage as the difference between the reference voltage for the first and last $\mathrm{pH} 7$ in the $\mathrm{pH}$ loop ( $\mathrm{pH} 7 \rightarrow 10 \rightarrow 7 \rightarrow 4 \rightarrow 7$ ). The hysteresis voltages for the TG, BG, and SG modes were 12.01, 74.30, and $115.10 \mathrm{mV}$, respectively. The drift rate is the change in reference voltage per hour, which results from the micro potential charge caused when ions penetrate the sensing membrane over a long period [23]. The drift rates in the TG, BG, and SG modes were 13.89, 60.39, and $109.36 \mathrm{mV} / \mathrm{h}$, respectively, as shown in Figure 8b. The sensing parameters of triple-gate a-IGZO TFT-based pH-ISFET sensor platform are summarized in Table 3. Compared to the TG mode, the BG mode has 18.7 times higher sensitivity, 6.2 times greater hysteresis voltage, and 4.3 times higher drift rate, while the SG mode has 42.5 times higher sensitivity, 9.6 times larger hysteresis voltage, and 7.9 times higher drift rate. This suggests that the SG mode has better stability and reliability than the other modes because its increases in hysteresis, voltage and drift rates are relatively low compared to its increase in sensitivity.

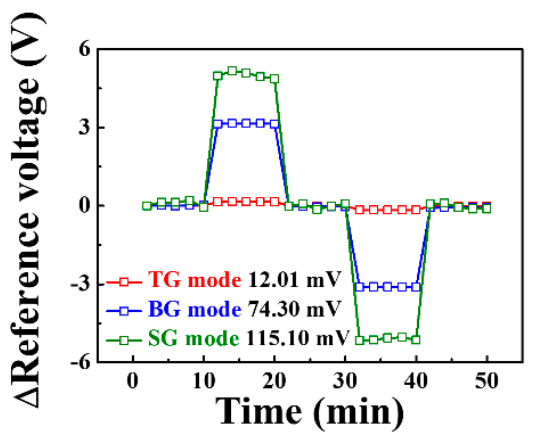

(a)

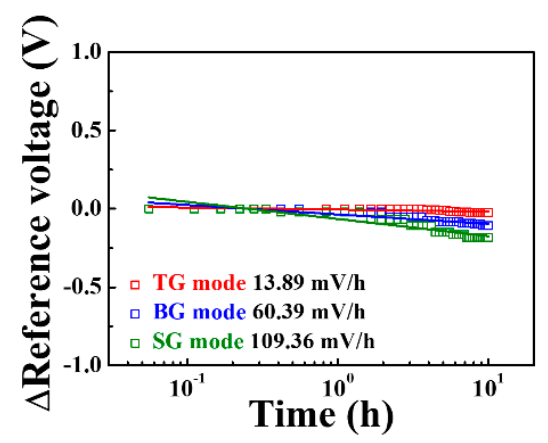

(b)

Figure 8. (a) Hysteresis voltage and (b) drift rate of the triple-gate a-IGZO TFT-based pH-ISFET sensor platform in the TG, BG, and SG modes.

Table 3. $\mathrm{pH}$ sensing parameters of the triple-gate a-IGZO TFT-based $\mathrm{pH}$-ISFET sensor platform in the TG, BG, and SG modes. Amplification refers to the amplified ratio of each mode relative to the TG mode.

\begin{tabular}{ccccccc}
\hline \multirow{2}{*}{ Operation Mode } & \multicolumn{2}{c}{ Sensitivity $(\mathbf{m V} / \mathbf{p H})$} & \multicolumn{2}{c}{ Hysteresis Voltage $(\mathbf{m V})$} & \multicolumn{2}{c}{ Drift Rate (mV/h) } \\
\cline { 2 - 7 } & Measured & Amplification & Measured & Amplification & Measured & Amplification \\
\hline TG & 55.6 & 1.0 & 12.0 & 1.0 & 13.9 & 1.0 \\
\hline BG & 1038.9 & 18.7 & 74.3 & 6.2 & 60.4 & 109.4 \\
\hline SG & 2363.9 & 42.5 & 115.1 & 9.6 & 7.9 \\
\hline
\end{tabular}

\subsection{Urea Sensing Characteristics of the Triple-Gate a-IGZO TFT-Based Urea-EnFET Sensor Platform}

After immobilization of the urease enzyme, we performed a control test to assess the selectivity of the urea-EnFET sensor platform and to verify whether it selectively detects the urease-urea interaction. In the case of the $\mathrm{SnO}_{2}$-urea interaction, there is no shift in the reference voltage, as shown in Figure 9a, but for the urease-urea interaction, a shift in reference voltage can be observed in Figure $9 \mathrm{~b}$. This indicates that the urease enzyme was immobilized on the $\mathrm{SnO}_{2}$ and that it reacted with urea, verifying the selectivity. Furthermore, to ensure the reliability and stability of the measurements, we measured the variation in the drain current over time after supplying various concentrations of urea electrolytes to the EG reservoir and measured the drain current-time in the SG mode while biasing a constant voltage $\left(\mathrm{V}_{\mathrm{SG}}: 15 \mathrm{~V}, \mathrm{~V}_{\mathrm{D}}: 1 \mathrm{~V}\right)$ for $300 \mathrm{~s}$. Figure $9 \mathrm{c}$ shows the measured response time of the urea-EnFET sensor platform for various concentrations of urea electrolytes ranging from pUrea 0.5 to $\mathrm{pUrea} 5$. The urease-urea interaction occurred for $120 \mathrm{~s}$ and reached equilibrium through diffusion of the end-product. This suggests that 
the proposed urea-EnFET sensor platform can reliably detect urea electrolyte in about $300 \mathrm{~s}$ after the urease-urea reaction is initiated. We therefore measured the transfer characteristic curves after $300 \mathrm{~s}$ of supplying urea electrolyte to the EG reservoir for stable measurement.

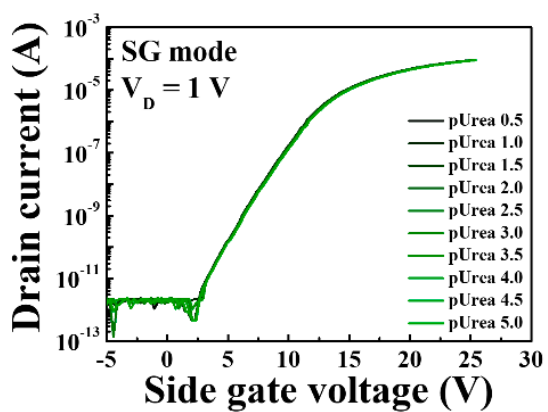

(a)

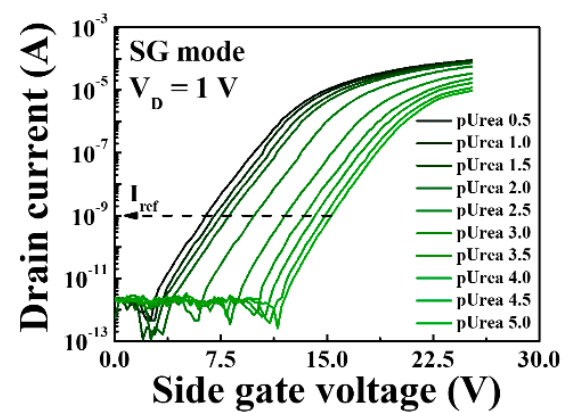

(b)

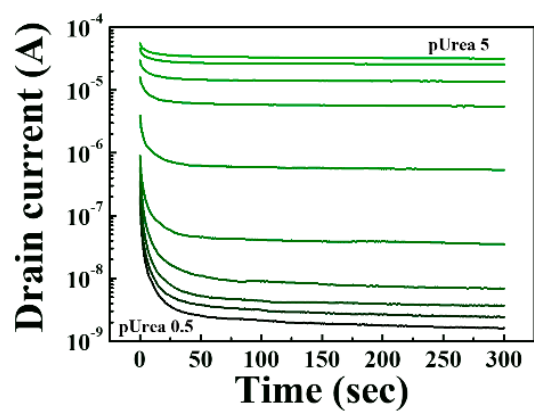

(c)

Figure 9. Transfer characteristic curves of the triple-gate a-IGZO TFT-based urea-EnFET sensor platform under the SG mode for (a) the interaction between the $\mathrm{SnO}_{2}$ membrane and urea and (b) the interaction between urease and urea (control test). (c) Drain current variation over time for various urea concentrations to confirm the response time needed to reach the equilibrium state of the reaction.

Figure 10 shows the sensing characteristics for the urease-urea reaction of the triplegate a-IGZO TFT-based urea-EnFET sensor platform in the TG, BG, and SG modes for various urea concentrations. To validate the triple-gate a-IGZO TFT-based urea-EnFET sensor platform, we detected urea for a wide range of urea concentrations: from pUrea 0.5 to pUrea $5(10 \mu \mathrm{M}$ to $316 \mathrm{mM})$ in PBS buffer solution. The transfer characteristic curves for the urea-EnFET sensor platform in the TG, BG, and SG modes as a function of urea concentration are shown in Figure 10a,b, and Figure 10c, respectively. The end-product of the urease-urea reaction decreases the surface potential of the $\mathrm{SnO}_{2}$ sensing membrane such that when the urea concentration increases, the surface potential decreases, and the reference voltage shifts in a positive direction. As a result, the shift of the transfer characteristic curve was larger in the BG mode than in the TG mode and greater in the SG mode than in the BG mode. Figure 10d shows the shift in the reference voltage in the TG, BG, and SG modes for various urea concentrations, as extracted from Figure 10a-c. The shift in the reference voltage due to the urease-urea reaction can be divided into three regions: (R1) a small change in the reference voltage for large urea concentrations from pUrea 0.5 to pUrea 2, (R2) the greatest change in the reference voltage from pUrea 2 to pUrea 3.5, and (R3) a small change in the reference voltage for low urea concentrations from pUrea 3.5 to pUrea 5. The low response in $\mathrm{R} 1$ is due to the excessive concentrations of $\mathrm{OH}-$ ions and by-products of the urease and urea reaction. At high $\mathrm{OH}^{-}$ion concentrations in the electrolytes, the urease enzyme exhibits lower reactivity toward urea. The high response in R2 is most useful for urea electrolyte detection because it results in a higher voltage shift, whereas the low voltage shift in R3 is due to the low urea concentration.

Figure 11 shows the urea sensitivity for the SG, BG, and TG modes of the triple-gate a-IGZO TFT-based urea-EnFET sensor platform in the R1, R2, and R3 regions extracted from Figure 10d. In R1, the urea sensitivity in the TG, BG, and SG modes was 33.2, 436.3, and $1272.7 \mathrm{mV} /$ pUrea, respectively. In R2, where the largest signal change was observed, the urea sensitivity in the TG, BG, and SG modes was 90.1, 1279.0, and $3632.1 \mathrm{mV} / \mathrm{pUrea}$, respectively. Finally, in R3, the urea sensitivity of the TG, BG, and SG modes was reduced to $45.3,441.5$, and $1051.0 \mathrm{mV} / \mathrm{pUrea}$, respectively. This result suggests that the urea sensitivity of the proposed urea-EnFET sensor platform can be amplified in the BG and SG modes similarly to the sensitivity amplification of the pH-ISFET sensor platform. We found that the SG mode is suitable for detecting urea electrolytes at a wide range of concentrations. Table 4 summarizes the characteristics of previously reported urea sensors for comparison with the proposed urea sensor. The urea-EnFET sensor fabricated in this study exhibits properties superior to those of the previously reported urea sensors, in particular, its 
exceptionally high sensitivity and linearity for a wide range of urea concentrations from pUrea 0.5 to 5 .

Table 4. Comparison of the analysis characteristics of the proposed urea-EnFET sensor platform and previously reported ISFET-based urea sensors.

\begin{tabular}{ccccc}
\hline Reference & Transducer & Sensitivity ( $\mathbf{m V / p U r e a )}$ & Range (pUrea) & Linearity (\%) \\
\hline [24] & N-channel Si MOSFET fabricated by ITE & 61.0 & $2-3.25$ & NA \\
\hline [25] & N-channel Si MOSFET CD4007UB & 62.4 & $2-3.25$ & 98.6 \\
\hline \multirow{3}{*}[26]{} & \multirow{3}{*}{ N-channel Si MOSFET CD4007UBE } & 43.9 & $0.5-2$ & NA \\
\cline { 3 - 4 } & & 109.0 & $2-3.5$ & NA \\
\cline { 3 - 4 } This work & \multirow{3}{*}{ Triple-gate a-IGZO TFT } & 9.8 & $3.5-5$ & NA \\
\cline { 3 - 4 } & & 1272.7 & $0.5-2$ & 98.2 \\
\cline { 3 - 4 } & & 3632.1 & $2-3.5$ & 99.0 \\
\hline
\end{tabular}

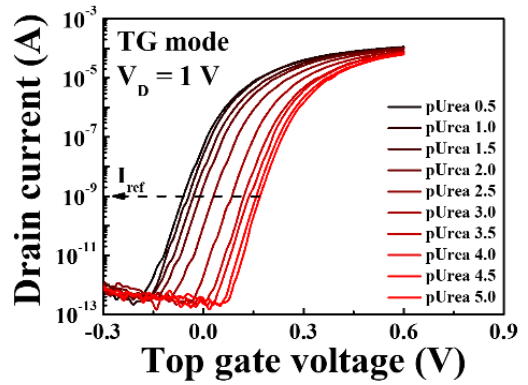

(a)

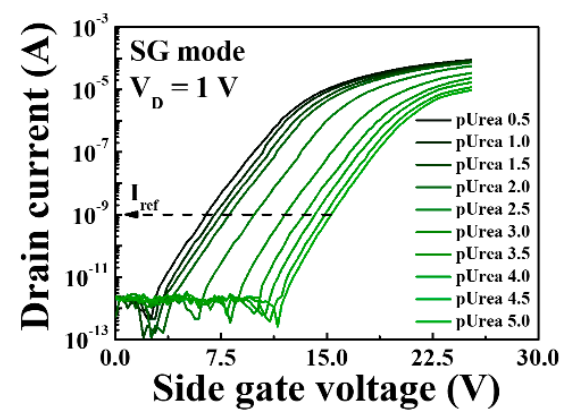

(c)

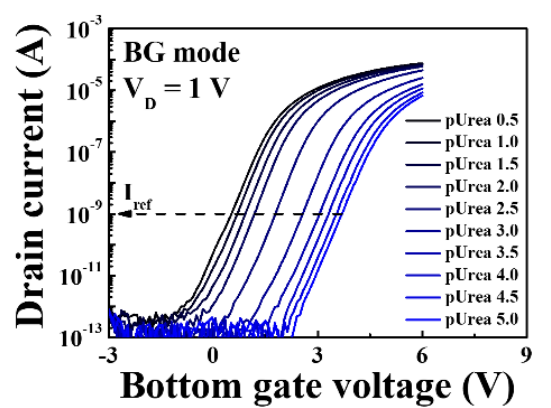

(b)

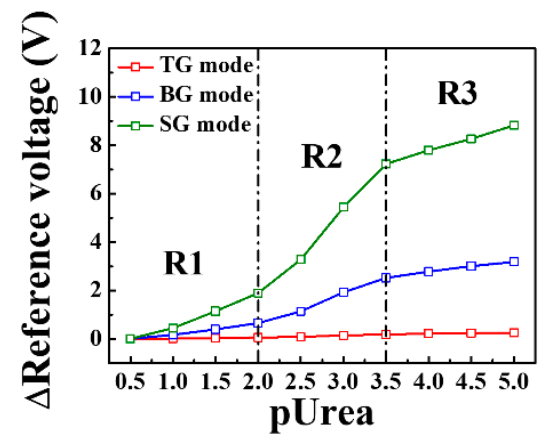

(d)

Figure 10. Transfer characteristic curves in urea-urea reactions of the triple-gate a-IGZO TFT-based urea-EnFET sensor platform in the (a) TG, (b) BG, and (c) SG modes for various urea concentrations. (d) Change in the reference voltage of the triple-gate a-IGZO TFT-based urea-EnFET sensor platform as a function of pUrea. 


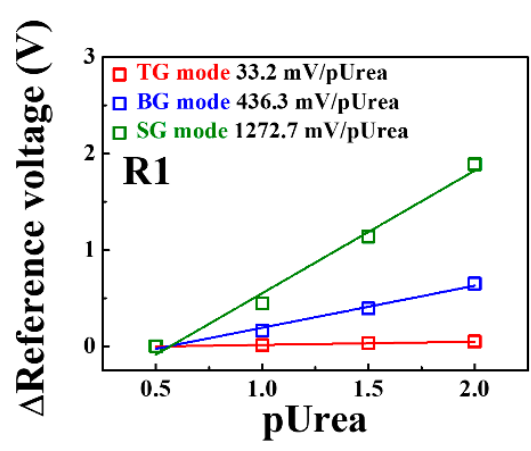

(a)

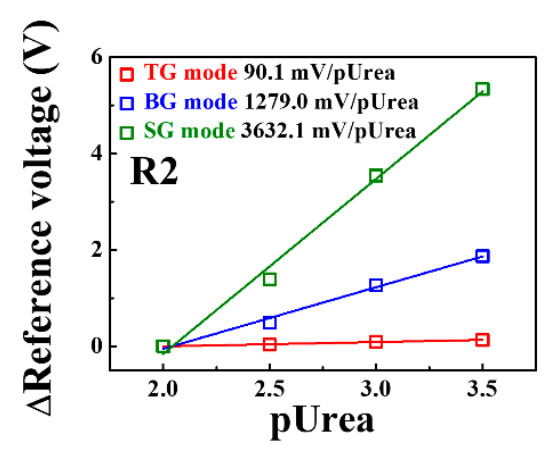

(b)

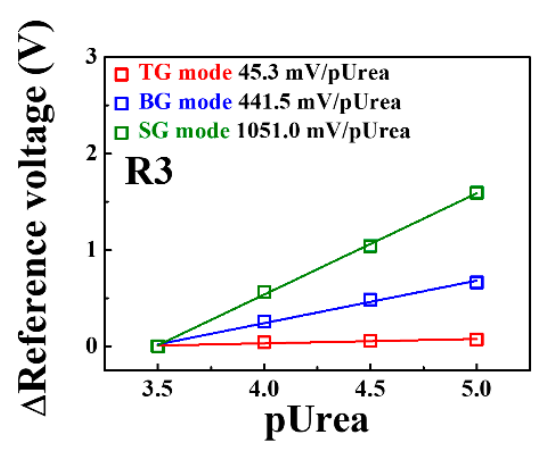

(c)

Figure 11. Change in the reference voltage for the TG, BG, and SG modes of the triple-gate a-IGZO TFT-based urea-EnFET sensor platform as a function of pUrea in the (a) R1, (b) R2, and (c) R3 regions.

\section{Conclusions}

In this study, we investigated a highly sensitive, transparent urea-EnFET based POC sensor using a triple-gate structure a-IGZO TFT. The urea-EnFET sensor consists of an $\mathrm{SnO}_{2}$ EG with immobilized urease for the sensing unit and a triple-gate a-IGZO TFT for the transducer unit. The proposed triple-gate a-IGZO TFT transducer has a TG at the top of the channel, a BG at the bottom of the channel, and a SG on the side of the channel, and it exhibits excellent sensitivity amplification for urea detection. In particular, in the SG mode, we were able to detect urea with a very high sensitivity of $3632.1 \mathrm{mV} / \mathrm{pUrea}$ in the pUrea range of 2 to 3.5 ; this is about 50 times larger than the sensitivity reported in previous works. Furthermore, high urea sensitivity and reliability were even obtained in the low pUrea range of 0.5 to 2 and high pUrea range of 3.5 to 5 . We therefore expect the proposed urea-EnFET sensor with a triple-gate a-IGZO TFT and $\mathrm{SnO}_{2}$ EG with immobilized urease to be a useful platform for POC diagnostic tests that require high sensitivity and high reliability.

Author Contributions: S.-K.C.: Conceptualization, Formal analysis, Methodology, Investigation, Data curation, Visualization, Software, Resources, Writing-Original Draft. W.-J.C.: Conceptualization, Methodology, Investigation, Resources, Formal analysis, Funding acquisition, Supervision, Validation, Writing-Review \& Editing. Both authors have read and agreed to the published version of the manuscript.

Funding: The present research was conducted by the Research Grant of Kwangwoon University in 2020, and this work was supported by the National Research Foundation of Korea (NRF) grant funded by the Korean government (MSIT) (No. 2020R1A2C1007586).

Conflicts of Interest: The authors declare no conflict of interest.

\section{References}

1. Zhang, J.; Liu, G.; Zhang, Y.; Gao, Q.; Wang, D.; Liu, H.J. Simultaneous determination of ethyl carbamate and urea in alcoholic beverages by high-performance liquid chromatography coupled with fluorescence detection. J. Agric. Food Chem. 2014, 62, 2797-2802. [CrossRef]

2. Qin, J.; Chao, K.; Kim, M.S. Simultaneous detection of multiple adulterants in dry milk using macroscale Raman chemical imaging. Food Chem. 2013, 138, 998-1007. [CrossRef]

3. Yang, Y.; Wang, Z.; Yang, M.; Guo, M.; Wu, Z.; Shen, G.; Yu, R. Inhibitive determination of mercury ion using a renewable urea biosensor based on self-assembled gold nanoparticles. Sens. Actuators B Chem. 2006, 114, 1-8. [CrossRef]

4. Huang, X.; Li, X.; Wang, Y.; Zhou, M. Effects of environmental factors on the uptake rates of dis-solved nitrogen by a salt-water green alga (Oocystis Borgei snow). Bull. Environ. Contam. Toxicol. 2012, 89, 905-909. [CrossRef] [PubMed]

5. Singh, M.; Verma, N.; Garg, A.K.; Redhy, N. Urea bio-sensors. Sens. Actuators B Chem. 2008, 134, 345-351. [CrossRef]

6. Chirizzi, D.; Malitesta, C. Potentiometric urea biosensor based on urease-immobilized by an electro-synthesized poly (ophenylenediamine) film with buffering capability. Sens. Actuators B Chem. 2011, 157, 211-215. [CrossRef]

7. Dhawan, G.; Sumana, G.; Malhotra, B.D. Recent developments in urea biosensors. Biochem. Eng. J. 2009, 44, 42-52. [CrossRef]

8. Bergveld, P. Development of an ion-sensitive solid-state device for neurophysiological measurements. IEEE. Trans. Biomed. Eng. 1970, 1, 70-71. [CrossRef] [PubMed] 
9. Waleed Shinwari, M.; Jamal Deen, M.; Landheer, D. Study of the electrolyte-insulator-semiconductor field-effect transistor (EISFET) with applications in biosensor design. Microelectron. Reliab. 2007, 47, 2025-2057. [CrossRef]

10. Jankovic, V.; Chang, J.P. HfO2 and ZrO2-based microchemical ion sensitive field effect transistor (ISFET) sensors: Simulation \& experiment. J. Electrochem. Soc. 2011, 158, 115-117.

11. Chi, L.L.; Chou, J.C.; Chung, W.Y.; Sun, T.P.; Hsiung, S.K. Study on extended gate field effect transistor with tin oxide sensing membrane. Mater. Chem. Phys. 2000, 63, 19-23. [CrossRef]

12. Lyons, C.; Clark, L.C., Jr. Electrode systems for continuous monitoring in cardiovascular surgery. Ann. N. Y. Acad. Sci. 1962, 102, 29-45.

13. Caras, S.; Janata, J. Field effect transistor sensitive to penicillin. Anal. Chem. 1980, 52, 1935-1937. [CrossRef]

14. Gonçalves, D.; Prazeres, D.M.F.; Chu, V.; Conde, J.P. Detection of DNA and proteins using amorphous silicon ion-sensitive thin-film field effect transistors. Biosens. Bioelectron. 2008, 24, 545-551. [CrossRef]

15. Castellarnau, M.; Zine, N.; Bausells, J.; Madrid, C.; Juarez, A.; Samitier, J.; Errachid, A. Integrated cell positioning and cell-based ISFET biosensors. Sens. Actuators B Chem. 2007, 120, 615-620. [CrossRef]

16. Soldatkin, A.P.; Montoriol, J.; Sant, W.; Martelet, C.; Jaffrezic-Renault, N. A novel urea sensitive biosensor with extended dynamic range based on recombinant urease and ISFETs. Biosens. Bioelectron. 2003, 19, 131-135. [CrossRef]

17. Bergveld, P. ISFET based enzyme sensors. Biosensors 1987, 3, 161-186.

18. Meixner, L.K.; Koch, S. Simulation of ISFET operation based on the site-binding model. Sens. Actuators B Chem. 1992, 6, 315-318. [CrossRef]

19. Park, J.K.; Jang, H.J.; Park, J.T.; Cho, W.J. SOI dual-gate ISFET with variable oxide capacitance and channel thickness. Solid-State Electron. 2014, 97, 2-7. [CrossRef]

20. Jang, H.J.; Bae, T.E.; Cho, W.J. Improved sensing performance of polycrystalline-silicon based dual-gate ion-sensitive field-effect transistors using high-k stacking engineered sensing membrane. Appl. Phys. Lett. 2012, 100, 253703. [CrossRef]

21. Jeon, J.H.; Cho, W.J. Triple Gate Polycrystalline-Silicon-Based Ion-Sensitive Field-Effect Transistor for High-Performance Aqueous Chemical Application. IEEE Electron Device Lett. 2019, 40, 318-320. [CrossRef]

22. Tsai, C.N.; Chou, J.C.; Sun, T.P.; Hsiung, S.K. Study on the sensing characteristics and hysteresis effect of the tin oxide pH electrode. Sens. Actuators B Chem. 2005, 108, 877-882. [CrossRef]

23. Jamasb, S.; Collins, S.; Smith, R.L. A physical model for drift in pH ISFETs. Sens. Actuators B Chem. 1998, 49, 146-155. [CrossRef]

24. Lue, C.E.; Yu, T.C.; Yang, C.M.; Pijanowska, D.G.; Lai, C.S. Optimization of urea-EnFET based on Ta2O5 layer with post annealing. Sensors 2011, 11, 4562-4571. [CrossRef]

25. Yang, C.M.; Wang, I.S.; Lin, Y.T.; Huang, C.H.; Lu, T.F.; Lue, C.E.; Pijanowska, D.G.; Hua, M.Y.; Lai, C.S. Low cost and flexible electrodes with NH3 plasma treatments in extended gate field effect transistors for urea detection. Sens. Actuators B Chem. 2013, 187, 274-279. [CrossRef]

26. Silva, G.O.; Mulato, M. Urea Detection Using Commercial Field Effect Transistors. ECS J. Solid State Sci. Technol. 2018, 7, Q3014-Q3019. [CrossRef] 